\title{
PROTON: A TRANSITION LAYER API
}

\author{
Dhruv, Sagar Kc, Gargi Shodangi, Aayush Soni \\ Manav Rachna International Institute of Research and Studies \\ Electronics and Communication Engineering (IOT) \\ New Delhi, India
}

\begin{abstract}
API (application programming interface): API act as a layer which helps software's communicate with computer hardware, Think of it as an instance where you go to a restaurant, You give order to waiter, waiter gives your order to chef in place, here you didn't have to go to chef directly and worry for waiting in line to give your order, and waiter did this thing for you while you were waiting at your table, same way while you use your computer without any complexity, Api acts as an middle man between your hardware and software and doing all the work
\end{abstract}

\section{Keywords-API, proton, vulkan, DirectX}

\section{INTRODUCTION}

Proton is a compatibility layer for Microsoft based x64 software's to run on Linux-Based operating systems. Proton is constantly being developed by valve and valve has released its code to public so developers, so people can constantly update its firmware, it includes several patches and libraries to improve performance and compatibility with windows based programs, proton incorporates several libraries that improve 3D object performance, these include direct 3D to Vulkan transition layers,

D9VK for Direct 3D 9

DXVK for Direct 3D 10\11

VKD3D for Direct 3D 12 (most recent development)

Being a fork of vine, Proton maintains a very similar compatibility with windows application as its upstream counterpart. In addition to official whitelist, a large portion of windows catalog is reportedly compatible, albeit unofficially with proton

\subsection{Proton DB}

Proton DB is a unofficial community that collects and updates crowd sourced data describing the compatibility of given title with proton on rating scale of "Borked" to "Platinum"

\subsubsection{Recent development in versions of proton}

Proton GE: build of proton with most recent release of vanilla vine, ffmpeg enabled for faudio by default, and all of proton patches ported over to applied to vine, as well as vine staging and vkd3d
Boxtron: It is a compatibility tool to run Dos games using native Linux DOSbox project was a sister project of luxtorpeda and Roberta and featuring lower input lag

Luxtrorpeda: same as boxtron but with added compatibility to work with newer game engines like unreal engine, Bethesda and unity 5 and added support for upcoming unreal engine 5

\section{PROPOSED ALGORITHM}

\section{Apple's metal to proton: A way out}

Metal is low level overhead hardware accelerated 3D graphic and computer shader Application programming interface (API), debuted in IOS 8, metal combines function similar to Open GL and Open CL under one API, it is an object-oriented API that can be invoked using swift or c programming languages, full blown GPU execution is controlled via metal shading languages, according to APPLE MSL is single, unified language that allows tighter integration between Graphics and compute programs, since MSL is c++ based ,its familiar to developers to work with

Metal started support for integrated gpu's from Intel since Intel hd 4000 graphics series, NVidia kepler based architecture 600 series most popular being $640 \mathrm{~m}$ being shipped in earlier models of MacBook pro's and AMD GCN based gpu,s like Radeon 7770 or 7970 as for now support upcoming Radeon 5000 series which is hybrid of RDNA 2 and GCN architecture 2.1 The catch?

Just like apple has metal for mac os, Microsoft have direct $\mathrm{x}$ and with its 20 year old head start, it is far more superior than metal and test shows valve based steam gaming titles and some productivity benchmarks direct $\mathrm{x}$ based machine outperforms metal based machine by a very large margin

\subsubsection{Here are some tests}

We used a hackintosh (installed mac os on custom built pc) -core i5 $84002.8 \mathrm{GHz}$ (6Core/6Thread)

- 16 GB ddr4 $2400 \mathrm{MHz}$ ram

-Radeon RX 5704 GB GGDR5

And a MacBook pro 2015 ' 13

-i5 5887u $2.9 \mathrm{GHz}$ (2core/2 thread)

-iris 6100 integrated graphics

- 8 GB ddr3 1333 MHz ram

We ran few games on each machines and observed the differencel

Total war saga 


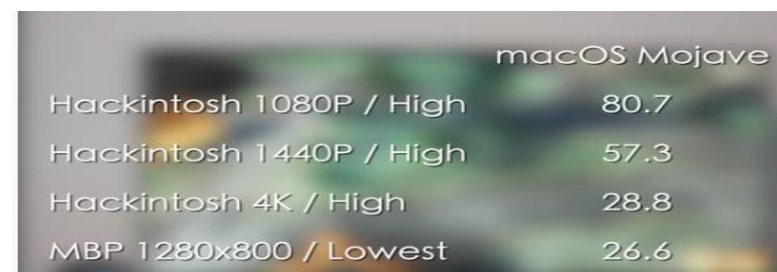

Rise of tomb raider hammer

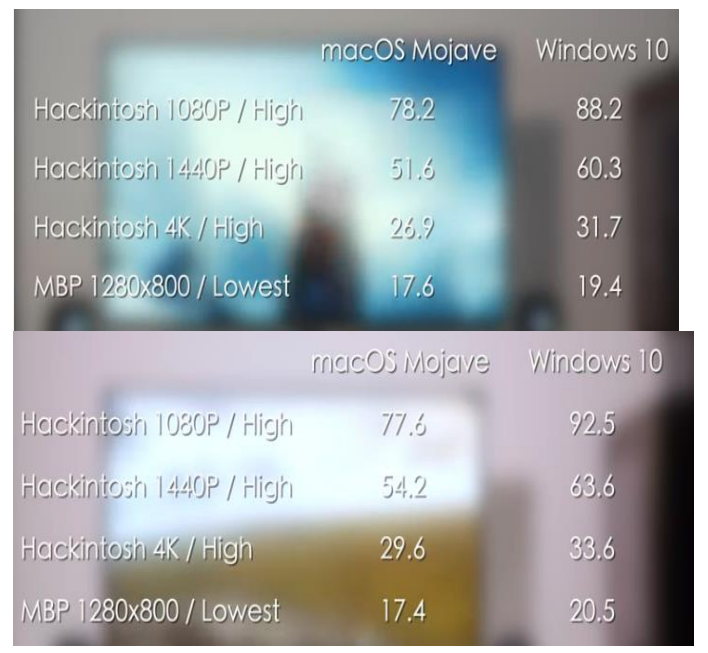

We also performed uniengine valley benchmark on our hackintosh

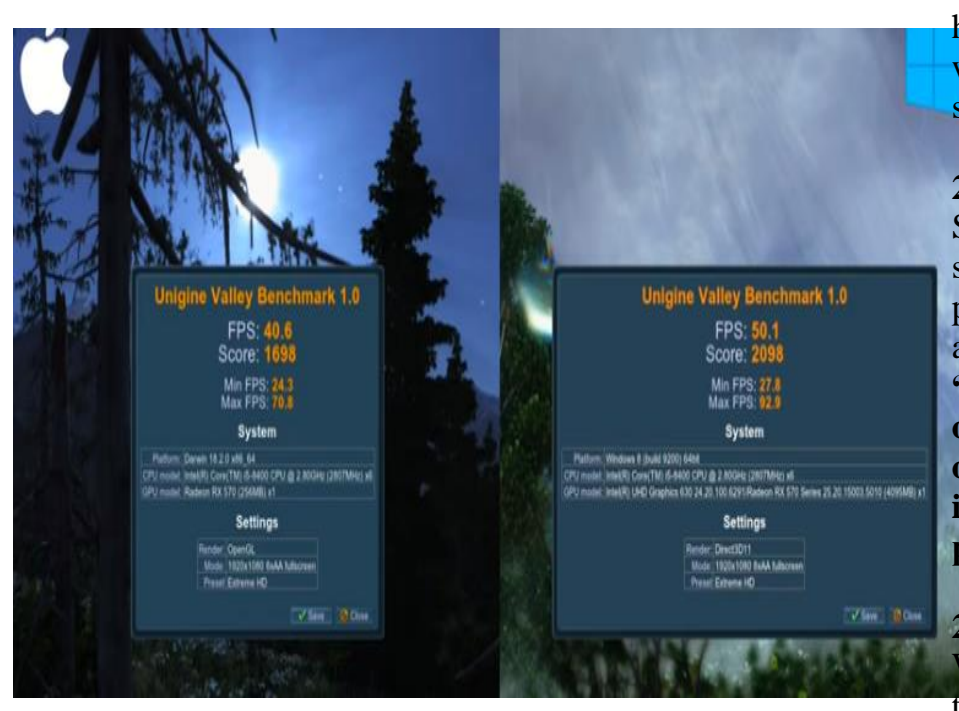

These test showed us that metal is far from beating direct and result of that game developers and adobe prioritize windows users above mac users, in result valve despite developers importing x86 based games to ARM for mobile devices and Nintendo switch with Tegra GPU, porting x86 applications to mac isn't worth to developer's because they would move a very few units because of politics played by apple when apple killed support for 32 bit applications on mac os Catalina back in 2018, and defending end of open GL as threat of their leaving developer's

81.9

68.2.1.2 What is proton play?

35. Indirectly steam play, valve have been developing it since 2015 , ince vine was a general level compatibility layer, proton-being fork of vine it is only focused on gaming and improving direct3D performance over vine, proton Is basically set of tools and compatibility layer that allows windows games to be ran on Linux using steam native Linux client, the direct 3D transition layers, since direct 3D is property of Microsoft windows, there is no implementation of this on Linux, these tools convert direct $\mathbf{x}$ calls into Vulkan

Steam recently have been employing developer of DXVK because its direct implementation of translation layer of D3D11 and they are also working on VKd3d with web weavers which is direct3D 12 implementation on Linux

\subsection{Native bridges}

Enables use of Linux native code like open VR and steam works to be accessed by game running through proton, which allows steam games to depend on steam compiled code, therefore cause no performance penalty to use these functions while improving support also added support for third party controllers, creating bridge between steam client controller interface and game to add support to more controllers than game originally supported, valve also funded development of DVK Also provided brand-new features of vulkan to mesa to help in development of new controls for Linux, they are also working with AMD,INTEL, and NVIDIA to work on driver support for their new gpu's

2.2.1 What steam had to say on proton steam play?

Steam encouraged developers to start enforcing Vulkan API, so even when developers didn't want to target Linux for their platform, they had choice to release on respective platform anytime here is there statement

"We recommend you to target vulkan natively in order to offer the best performance possible on all platforms, or offer it at least as an option. Its good idea to avoid any invasive third party DRM middleware, as they sometimes prevent compatibility features from working as intended"

\subsubsection{Problem with MAC OS}

While OPEN GL still carries its support to mac os, there is no telling when apple will remove the plug and it troubles indie game Developer's and it begs the question Andy wood creator of titles like F1, rise of tomb raider, sleeping dogs, made this statement

"It's been a challenge to work with Open GL for some years now, especially with advent of new graphics technologies for example we weren't able to release F1 2015 on Mac os because Open Gl didn't have necessary features"

Steam helping apple but why? 


\section{International Journal of Engineering Applied Sciences and Technology, 2020 \\ Vol. 5, Issue 7, ISSN No. 2455-2143, Pages 303-307 \\ Published Online November 2020 in IJEAST (http://www.ijeast.com)}

Apple and valve share a common rival that is Microsoft, with release of Windows $10 \mathrm{~s}$ and Windows $10 \mathrm{x}$ that Microsoft wants to take an apple like walled garden approach by pushing software sales through Microsoft store which jeprpodize Valves business, to defend this valve released Proton a compatibility layer between direct 3D and vulkan based on vine

\section{METHODOLOGY}

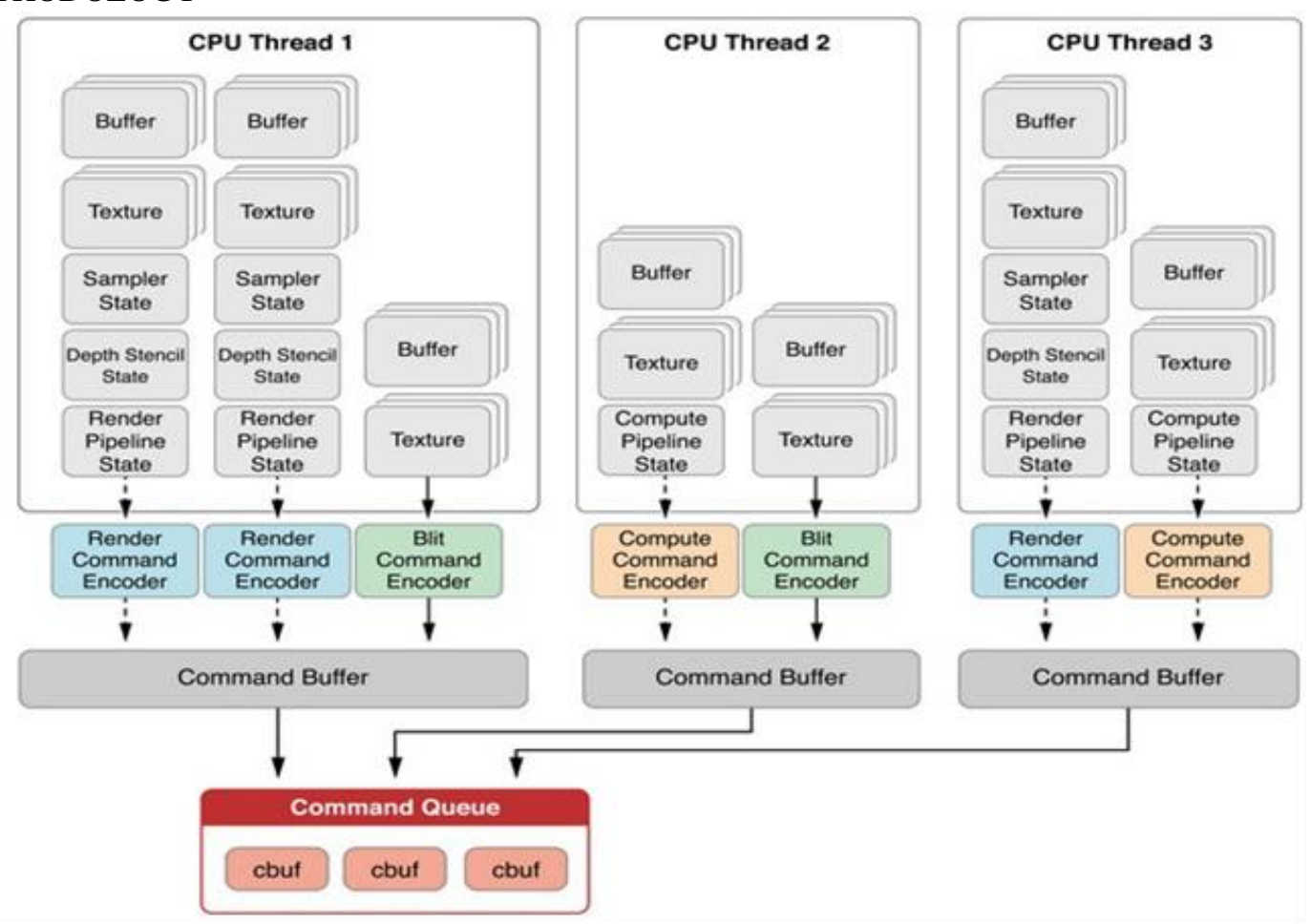

FIG 1. Metal pipeline state overhead

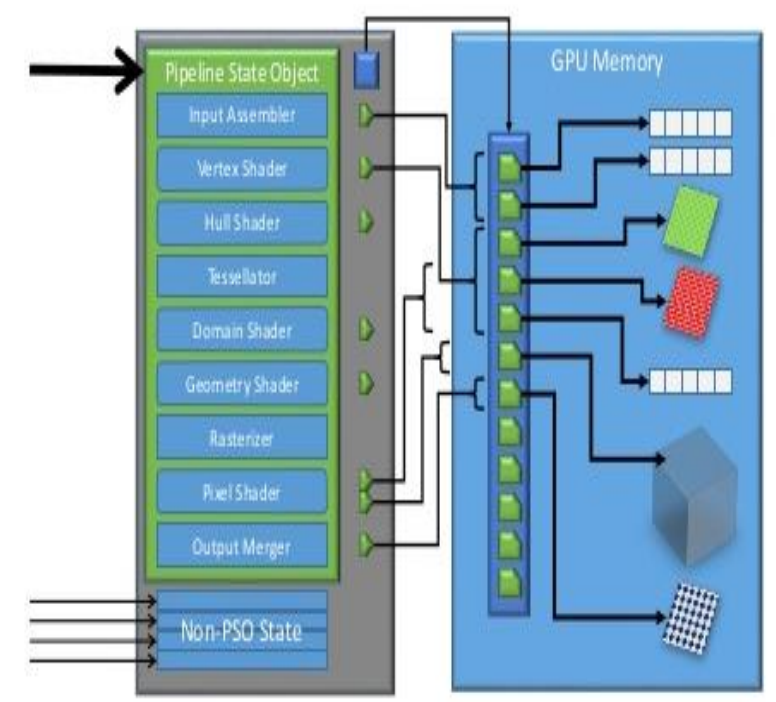

\subsection{Rendering pipelines}

A conceptual $3 \mathrm{~d}$ modal that describes what steps a system has to take to render a scene in $3 \mathrm{D}$ once $3 \mathrm{D}$ scene is rendered, graphic pipeline work is to turn that rendered $3 \mathrm{D}$ modal into what computer displays

\subsubsection{Conceptual framework of rendering pipeline of metal}

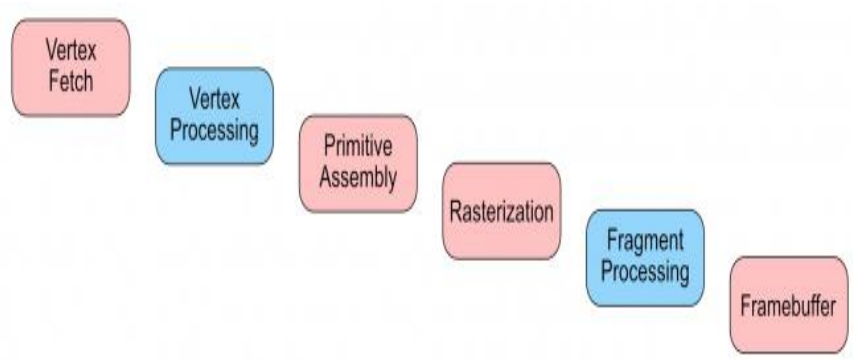

FIG 2. Direct x 12 pipeline state overhead 


\section{International Journal of Engineering Applied Sciences and Technology, 2020 \\ Vol. 5, Issue 7, ISSN No. 2455-2143, Pages 303-307 \\ Published Online November 2020 in IJEAST (http://www.ijeast.com)}

\subsubsection{Conceptual framework of rendering pipeline of direct}

\section{$\mathbf{X}$}

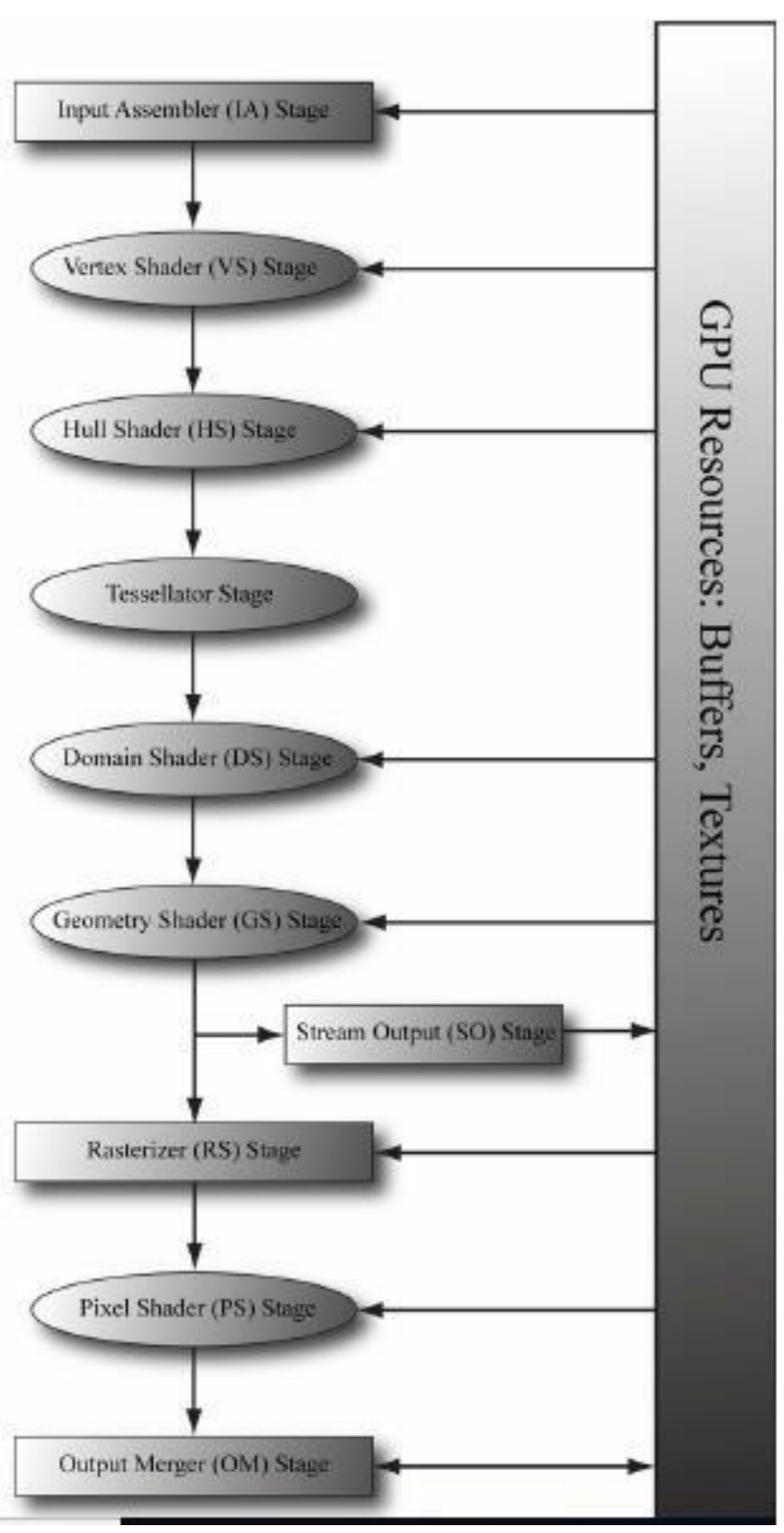

\subsection{Difference between the two?}

In terms of normal map, the difference results in how green channel of RGB texture, should be interpreted, Metal expects first pixel to be present at bottom while direct $\mathrm{x}$ expects it to be at the top, game developers invert the green channel of top of the normal map and generally prefer top down Y- approach of Direct $x$

Other differences include:

\begin{tabular}{lcl}
\hline Feature: & metal & DirectX \\
Vertex Blending & N/A & Yes \\
Multiple Operating Systems & Yes & No \\
Extension Mechanism & Yes & Yes \\
Development & apple & Microsoft \\
Thorough Specification & Yes & No \\
Two-sided lighting & Yes & No \\
Volume Textures & Yes & No \\
Hardware independent Z-buffers & Yes & Yes \\
Accumulation buffers & Yes & no (present in directr x 12) \\
Full-screen Antialiasing & Yes & Yes (dlsst txaa added) \\
Motion Blur & Yes & Yes \\
Depth of field & Yes & Yes (temporal) \\
Stereo Rendering & Yes & No \\
Point-size/line-width attribute & Yes & No \\
Picking & Yes & No \\
Parametric curves and surfaces & Yes & No \\
Cache geometry & display Lists & Vertex Buffers \\
System emulation & Hardware not present & Let app determine \\
Interface & Procedure calls & coM \\
Updates & Yearly & Yearly \\
Source Code & Sample & SDK Implementation \\
& &
\end{tabular}

\section{Apple's initiative for metal}

\subsection{Metal Stepping into the proton shoes}

"In recent times metal pay opened SDK to developers to integrates its native wallet"

At the same time apple releasing its native wallet SDK's, metal has opened its integration with proton, enabling developers to access functionality including ability to manage accounts on proton chain, after creating an account using registered api key, developers can directly connect to proton chain or test net, which allows for ios applications to be created when used in conjunction with SDK

The range of possibilities afforded by metal pay's SDK and API are extensive, allowing for creation of more smart and secure crypto wallet with controls for key management, wired transactions, handling ESR requests

Developers are being encourages to build test projects on proton chain and metal team thrives for third party developers to utilize these tools to create new applications built on apple pay's robust framework 


\section{International Journal of Engineering Applied Sciences and Technology, 2020 \\ Vol. 5, Issue 7, ISSN No. 2455-2143, Pages 303-307 \\ Published Online November 2020 in IJEAST (http://www.ijeast.com)}

\section{CONCLUSION}

Just like metal pay integrated with proton allows third party developers to utilize the apple's framework for making apps in both ios and android, and steam providing proton play to steam library to LINUX OS, only points to apple teaming up with Valve to connect this transition layer of proton (which is already working between Linux and windows) to work with metal in mac os, because metal is built on Open GL framework which is very old and outdated and if apple lets a transition layer like proton to let users play games or use applications directly using microsoft's already better direct X libraries and framework, it can revolutionize how consumers and enthusiast look mac os, which will force apple and Microsoft being even, so there will be more support more applications which users will have freedom to play or work on any operating system either Microsoft windows or apple's mac os without any significant performance loss and more competition is always good for a user

\section{REFERENCE}

[1].https://docs.substance3d.com/bake for difference between normal formats of API comparing open GL and direct $\mathrm{x}$ on basis of their referencing formats

[2].https://www.raywenderlich.com/5493-metal-renderingpipeline-tutorial for metal pipeline pictograph

[3].https://www.tutorialspoint.com/directx/directx_graphics_pi peline for DirectX pipeline overhead diagram

[4].https://alain.xyz/blog/comparison-of-modern-graphicsapis\#pipeline for understanding difference between different libraries different API use

[5].https://www.wisegeek.com/ for in-depth code analysis

[6].https://www.techworm.net/2020 for apple wallet analysis 